\title{
Positron Annihilation in Medical Substances of Insulin
}

\begin{abstract}
R. Pietrzak and R. Szatanik
Institute of Physics, Opole University, Oleska 48, 45-052 Opole, Poland

Positrons lifetimes were measured in medical substances of insulin $\mathrm{hu}$ man and animal), differing as far as the degree of purity and time of their activity in the organism are concerned. In all of the cases the spectrum of positron lifetime was distributed into three components, with the long-life component ranging from 1.8 to 2.08 ns and the intensity taking on values from 18 to $24 \%$. Making use of Tao-Eldrup model, the average radius of the free volume, in which $o$-Ps annihilated, and the degree of filling in the volume were determined. It was found that the value of the long-life component for human insulin is higher than that of animal insulin. Moreover, the value of this component clearly depends on the manner of purification of the insulin. It was also noticed that there occurs a correlation between the value of this component and the time after which it begins to be active in the organism, as well as the total time of its activity.
\end{abstract}

PACS numbers: $78.70 . \mathrm{Bj}$

\section{Introduction}

Insulin is a polypeptide hormone of the following global formula CHNOS, formed from two peptide chains A and B linked by two disulfide bridges. Chain A consists of 21 aminoacids and a disulfide bridge binding two particles of cistern (A6Cys-A11Cys). Chain B consists of 30 aminoacids. In a solution, insulin shows a quarternary structure, forming particles composed from 2,6 , and 8 chains.

Insulin analogues are also made use of in medicine, as they are products of a chemical modification of the insulin particle, for instance through an exchange of two aminoacids (e.g. Insulin Ispro - Humalog). Insulin changed in this way does not form hexameters, which causes it to work much faster and stronger, yet for much shorter time.

Medical substances of insulin are divided according to the following:

a) their origin (human or animal); 
b) degree of purification (e.g. highly purified by method of genetic engineering, chromatographically purified, highly purified, one-stage purified);

c) time of working (e.g. short working of medium and maximally prolonged time of working).

The pig-derived insulin differs from the human type with one aminoacid and the cow-derived type — with three aminoacids.

The working time of insulin is connected with its absorption from the subcutaneous tissue: the longer the given substance is absorbed, the slower it starts to work and the longer the total time of working of the medicine becomes. Prolonging the absorption of the medicine from the subcutaneous tissue is obtained through adding substances which retard absorption of insulin to insulin substances (e.g. protamines, zinc or their components).

Positrons lifetimes in 6 selected substances of insulin applied in medicine were investigated. They differed as for their origin (human and animal-derived), method of purification and working in human organism. The choice and properties of the substances are shown in the Table.

TABLE

Medical properties of examined parameters of insulin.

\begin{tabular}{l|c|c|c|c|c}
\hline \hline $\begin{array}{c}\text { Name of } \\
\text { preparation } \\
\text { diagrams }\end{array}$ & $\begin{array}{c}\text { No. on the } \\
\text { Type }\end{array}$ & $\begin{array}{c}\text { Method of } \\
\text { obtaining } \\
\text { Humalog }\end{array}$ & $\begin{array}{c}\text { hime after } \\
\text { which the } \\
\text { insulin takes } \\
\text { effect } \\
t_{0}[\mathrm{~h}]\end{array}$ & $\begin{array}{c}\text { Total time } \\
\text { of working } \\
t_{\mathrm{t}}[\mathrm{h}]\end{array}$ \\
\hline Humulin R & 2 & human & $\begin{array}{c}\text { Recombining } \\
\text { DNA }\end{array}$ & 0.25 & 4 \\
\hline Mixtard & 3 & human & $\begin{array}{c}\text { Isophane, highly } \\
\text { purified }\end{array}$ & 2 & 14 \\
\hline Humulin N & 4 & human & $\begin{array}{c}\text { Isophane, } \\
\text { biosynthesized }\end{array}$ & 2.5 & 18 \\
\hline Actruphene & 5 & human & Biosynthesized & 1 & 8 \\
\hline Lente & 6 & animal & Highly purified & 2 & 24 \\
\hline
\end{tabular}

\section{Experimental}

Solutions of insulins applied in medicine, of concentration $100 \mathrm{mg} / \mathrm{ml}$, were placed in two plexiglass vessels of $5 \mathrm{~mm}$ deep and $1.5 \mathrm{~cm}$ in diameter. They 
were covered with kapton foil of surface density of $0.9 \mathrm{mg} / \mathrm{cm}^{2}$, between which, in the "sandwich" geometry, there was a source of $\beta^{+}$radioactive isotope ${ }^{22} \mathrm{Na}$, covered with the above-mentioned kapton foil. The spectra of positron lifetime was obtained by means of a standard fast-slow spectrometer of resolution of $250 \mathrm{ps}$.

It was possible - with a good result - to resolve all the obtained positron lifetime spectra into three components ( $\chi^{2}$ close to 1$)$, while the component of the highest lifetime took on values of the nanosecond order. While interpreting results of measurements of positrons lifetimes, component $\tau_{1}$ is generally attributed to annihilation of para-Ps, and component $\tau_{2}$ - to free annihilation. Still, the most interesting, while interpreting the results, are the changes in the long-life component $\tau_{3}$ of values of the nanosecond order, which are attributed to annihilation of ortho-Ps in a pick-off process.

\section{Results}

Results of the research are presented in Figs. 1-4. Figure 1 shows a diagram of components of positron lifetime spectra for the examined types of insulin. As it can be seen, the values of components $\tau_{1}$ and $\tau_{2}$, within the limits of error, take on the same values for the majority of examined substances, like in the case of their intensities. The substance of insulin known under the trade name Humulin $\mathrm{N}$ is an exception here (marked as No. 4): the values $\tau_{1}$ and $\tau_{2}$ are considerably greater for it than for the other substances. The intensities of these components also markedly take on other values than those for the other substances of insulin. Much greater differences can be observed between the values of the long-life component
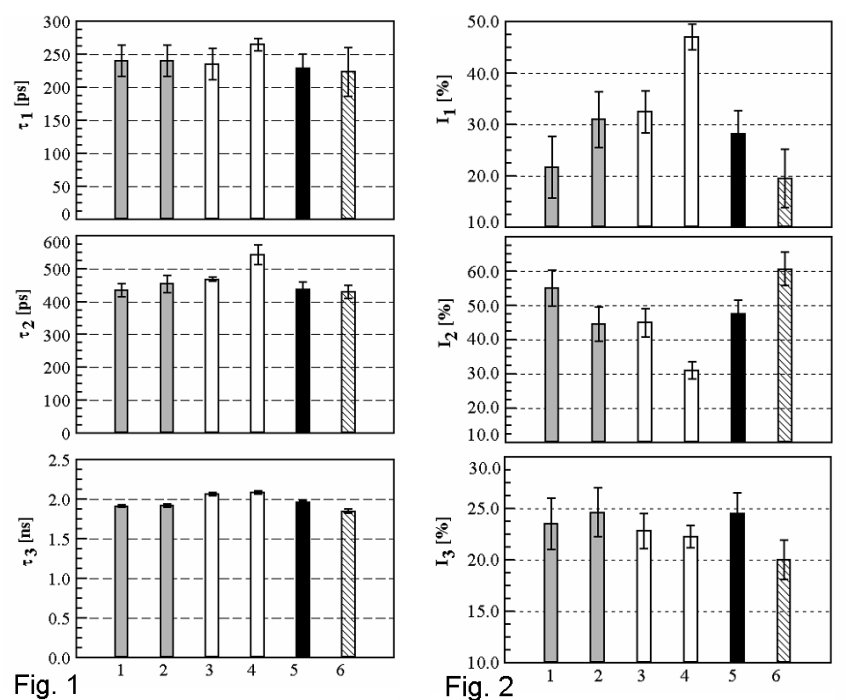

Fig. 1. Diagrams of positron lifetime components in investigated insulins.

Fig. 2. Diagrams of intensities of positron lifetime components in investigated insulins. 

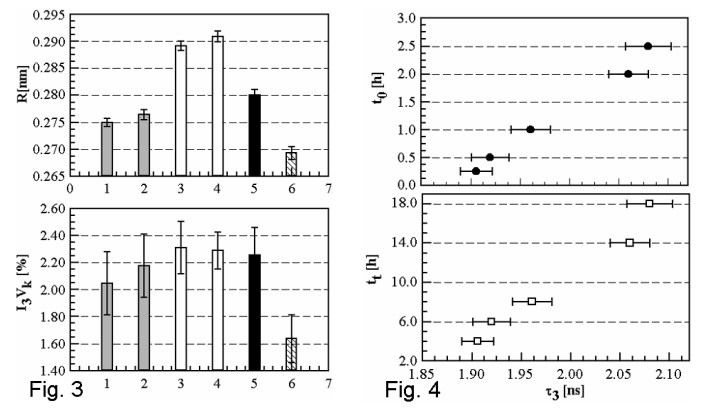

Fig. 3. Diagrams of average radius of the free volume $R$ and the product $I_{3} \cdot V_{k}$ in investigated insulins.

Fig. 4. Correlations between: time $t_{0}$ - after which substance begins act in the organism, total time acting of substance $t_{\mathrm{t}}$ and values of component $\tau_{3}$ of positron lifetime spectra - for human insulins only.

for different substances. Accordingly, for fast-reacting substances of the human insulin, obtained by means of DNA (Nos. 1 and 2), the values of component $\tau_{3}$ remain the same within the limits of error. Adding a substance retarding absorption of insulin by the organism - protamin - to the substance causes lifetimes of ortho-Ps (the so-called isophane insulins (Nos. 3 and 4)) to grow in a visible way. It is also evident that the long-life component for animal-derived insulin (Lente, No. 6) takes on clearly lower values, both as regards lifetime $\tau_{3}$ and its intensity $I_{3}$ (Fig. 2). It is hard to determine whether these differences are caused by the structure of the molecule of insulin, or, perhaps, by the presence of another substance which retards its absorption by the organism - in this particular case — zinc.

The mean lifetime of long-life component $\tau_{3}$ is connected with sizes and shape of traps in which the ortho-Ps found itself before annihilation, while its intensity - with their concentration. Tao and Eldrup [1,2] together with their co-workers proposed a model which combines the mean lifetime of $\tau_{3}$ with the mean radius of spherical empty space $R$ in solids within 0.25 to $16 \mathrm{~nm}$

$$
\tau_{3}=\lambda_{0}\left(1-\frac{R}{R+\Delta R}+\frac{1}{2 \pi} \sin \frac{2 \pi R}{R+\Delta R}\right)^{-1}
$$

where $\lambda_{0}^{-1}=0.5 \mathrm{~ns}, \Delta R=0.1656 \mathrm{~nm}$.

Equation (1) allows combining the mean radius of free volume with relative free volume, with the provision that the latter is defined as follows:

$$
V_{\mathrm{f}}=\frac{\left(V-V_{0}\right)}{V}
$$

where $V$ is the macroscopic volume of the solution, $V_{0}$ is the volume occupied by molecules of the solution. Wang et al. [3], as well as Kobayashi et al. [4] proposed a semi-empirical formula that allows determining the relative free volume on the 
basis of measurements of positron lifetime:

$$
V_{\mathrm{f}}=A I_{3} V_{k},
$$

where $I_{3}$ is the relative intensity of long-life component, $V_{k}=\frac{4}{3} \pi R^{3}$ is the volume of single spherical hole, $A$ - the normalization constant.

Figure 3 shows diagrams of the values calculated from Eqs. (1) and (3): average radius of the free volume $-R$ and the product $I_{3} \cdot V_{k}$ proportional to $V_{\mathrm{f}}$ for investigated substances. It can be seen that these values take on the least sizes for animal-derived insulin (Lente) and among human-derived insulin - for insulin prepared by DNA recombination method but maximum values - for isophane insulin.

Correlations between medical parameters of insulin substances and annihilation parameters of ortho-Ps have been investigated. Figure 4 shows clearly correlations between: time $t_{0}$ - after which substance begins act in the organism, total time acting of substance $t_{\mathrm{t}}$, and values of component $\tau_{3}$ of positron lifetime spectra. Dependences between values of intensity $I_{3}$ of the long-life component have not been found.

A full explanation of all the aspects relating to the presented results requires doing additional research.

\section{Conclusions}

- Values of times and intensities of short-life components of positrons lifetimes spectra $\left(\tau_{1}\right.$ and $\tau_{2}, I_{1}$ and $\left.I_{2}\right)$ in the investigated substances of insulin are practically the same.

- The lifetime of ortho-Ps in the investigated substances clearly depends on the type of insulin (human or animal-derived), method of obtaining and kind of admixtures influencing the speed of their absorption by the organism.

- There exists a correlation between medical parameters of the examined insulins and ortho-Ps lifetime in them.

\section{References}

[1] S.J. Tao, J. Chem. Phys. 56, 5499 (1972).

[2] M. Eldrup, D. Lightbody, J.N. Sherwood, J. Chem. Phys. 63, 51 (1981).

[3] I.I.Y.Y. Wang, H. Nakanishi, Y.C. Jean, T. Sandreczki, J. Polym. Sci. Part B, Polymer Physics 28, 1431 (1990).

[4] Y. Kobayashi, W. Zheng, E.E. Meyer, Y.D. Mc Gervey, A.M. Jamieson, R. Simha, Macromolecules 22, 2302 (1989). 